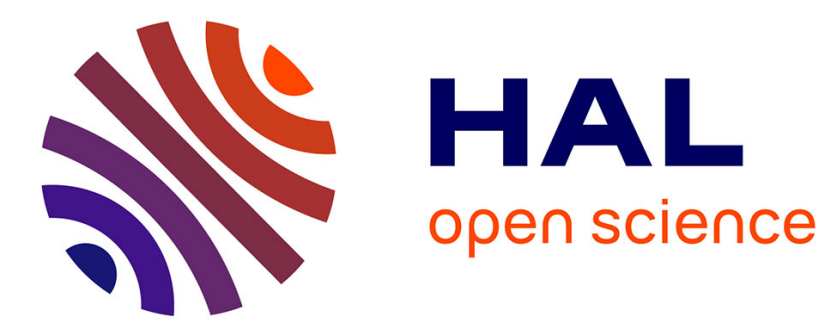

\title{
A two-phase approach for periodic home health care planning
}

Nizar Triki, Thierry Garaix, Xiaolan Xie

\section{To cite this version:}

Nizar Triki, Thierry Garaix, Xiaolan Xie. A two-phase approach for periodic home health care planning. 2014 IEEE International Conference on Automation Science and Engineering (CASE), Aug 2014, Taipei, Taiwan. pp.518 - 523, 10.1109/CoASE.2014.6899375 . emse-01108959

\section{HAL Id: emse-01108959 https://hal-emse.ccsd.cnrs.fr/emse-01108959}

Submitted on 4 Jan 2016

HAL is a multi-disciplinary open access archive for the deposit and dissemination of scientific research documents, whether they are published or not. The documents may come from teaching and research institutions in France or abroad, or from public or private research centers.
L'archive ouverte pluridisciplinaire HAL, est destinée au dépôt et à la diffusion de documents scientifiques de niveau recherche, publiés ou non, émanant des établissements d'enseignement et de recherche français ou étrangers, des laboratoires publics ou privés. 


\title{
A two-phase approach for periodic home health care planning
}

\author{
Nizar Triki ${ }^{1}$, Thierry Garaix ${ }^{1}$ and Xiaolan $\mathrm{Xie}^{1}$
}

\begin{abstract}
In this paper, we study the problem of periodic vehicle routing encountered in Home Health Care (HHC). The problem can be considered as a Periodic Vehicle Routing Problem with Time Windows (PVRPTW). It consists in establishing a planning of visits to patients over a given time horizon so as to satisfy the adherence to the care plan while optimizing the routes used in each time period. One two-stage mathematical formulation of this problem is proposed. We then propose a Tabu Search (TS) and a MIP-based Neighborhood Search method to compute the weekly and daily plan, respectively. These approaches are tested on large size instances. Keywords - Home health care, periodic vehicle routing, matheuristic.
\end{abstract}

\section{INTRODUCTION}

Home Health Care services are becoming increasingly important. Patients receive medical treatments at home. In France, two different types of structure exist. The first type, denoted HHC, focuses this study and is devoted to high qualification cares. The second type covers light care services. HHC are characterized by low flexibility in the adherence to the care plan and a high turn-over rate - the average length of stay is two weeks. HHC allow to reduce crowding in hospitals, some costs saving for health financial support, and better living conditions for many patients. The planning problem addressed here, and called Home Health Care Problem (HHCP), consists in assigning caregivers to jobs such that several exploitation costs are optimized, while a number of legal working (the use of extra working time is limited) and quality of service constraints are satisfied. The decision process is divided into two steps, the weekly plan and the daily plan. At the beginning of each week, known tasks are scheduled satisfying constraints of their care plan of the week. Hence, HHCP encapsulates a Periodic Vehicle Routing Problem with Time Windows, where day and time of visits are decided. The time windows arise from specific cares or regularity constraints on service times. Every day the daily plan has to be updated as new demands arise. Thus, a variant of the classical Vehicle Routing Problem (VRP) as to be solved preserving main characteristics of the current solution. Main characteristics to keep are, the date (day and time) of visits to patients, and the starting and ending times of caregivers' rounds. Logistics tasks as furniture or drugs delivery can be performed by any employee, logistician, nurse or nursing auxiliary. It is therefore interesting to integrate all types of employee in the same planning solution.

We propose in this paper one solving approach for each decision stage. We try to tight the gap between weekly

Center for Biomedical and Healthcare Engineering (LIMOS UMR CNRS 6158, ROGI) École Nationale Supérieure des Mines de Saint-Étienne. \{triki,garaix, xie\} @emse. fr planning and daily planning, by inclusion of an accurate evaluation of travel times in the weekly decision stage by considering a specific periodic vehicle routing problem. We also test the performance of an a priori robustness criterion based on the expected number of new tasks admitted each day.

The paper is organized as follows. In Section II we review the literature related to home health care problem and related vehicle routing problems. We describe in Section III the specific planning problem we address. The weekly and daily problems are presented in Section IV and Section V with their dedicated solution algorithm, respectively. Computational experiments are described and discussed in Section VI. We close the paper with a contribution of the work and suggestions for future research in Section VII.

\section{LITTERATURE REVIEW}

A large part of the literature addressing home health care planning focuses on the daily routing problem. This problem is modeled as a vehicle routing problem with or without time windows. In [1] an efficient column generation approach has been designed and applied to real-world data. In [2] the authors use a combination of linear programming, constraint programming, and metaheuristic.

Several works interested in middle-term planning of HHC services and dynamic insertion of new demands do not directly include the routing part of the workload. At this stage routing time is generally roughly approximated [3], [4] and [5]. In [6], the authors assigned weekly schedules to therapists to already fixed appointments.

The integration of traveling workload relates HHCP to Periodic Vehicle Routing Problem (PVRP), which is not usually associated with applications of HHC service. In a recent survey [7] on PVRP, most efficient heuristics and exact approaches are enumerated. Most efficient exact methods are derived from the algorithm proposed in [8]. Tabu search algorithms provide good results, but more sophisticated heuristics ([9]), like Variable Neighborhood Search ([10]) and genetic algorithms ([11]) are able to improve these results. Its dynamic version is treated in [12] and [13]. In the first work, a myopic approach is developed. A twophase approach is used in the second work, where the routes are roughly approximated with an aggregated measure and routes are considered in a daily re-optimization. In the closest problem [14] to ours, the number of resources that visit a patient during the day. The authors consider a periodic vehicle routing problem combining a resource rostering problem, where the objective is the continuity of care along he week. To find a first solution, they apply an 
algorithm based on constraint programming and then calls an adaptive large neighborhood search metaheuristic to improve the results.

Some of other papers related to weekly scheduling. In [15], authors proposed an Integer Linear Programming models which use the concept of patterns, i.e. a priori scheduling profiles, to combine the diverse decision levels. Their results on real instances show that pattern generation policies are crucial to address scheduling and routing in large Home Care instances.

A combination of the following characteristics of our approach make the contribution of this work: (1) the routing decisions at the weekly decision level; (2) an a priori anticipated daily workload; (3) the skills of employees; (4) the minimization of the deviation between tactical and operational solutions. The algorithm developed for the first stage decision level exploits a special case of PVRP, where each possible pattern - a list of days for visits - for a customer is a single day. Actually, customers in the PVRP are task of the HHCP instead of patients.

\section{PROBLEM DESCRIPTION}

The care plan usually repeats a weekly pattern. This pattern is evaluated and can be updated by the medical staff every week. Then, planning are built on a weekly base. Employees can call patients to fix appointments, order drugs or materials early. Some services have to be delivered within time windows because of medical requirements or patients' preferences. As most of stays are short - less than two weeks - the continuity of care (in the sense of a regular caregivers for each patient) is not required and caregiver assignment to tasks is only submitted to ability constraints.

High turn-over also implies that many demands are revealed along the week. New demands are mainly related to new admissions, but current patients can also require additional cares. An optimized weekly plan has to make easier new demands insertion, observing daily workload minimization for every employee. Although the care plan may allow some flexibility on the day of service, already fixed appointments can not be postponed to another day. Indeed, the change of a service day imply to get the agreement of the patient. Because of this validation step, it can not be integrated in the optimization model. Thus, on the eve of each day, the final daily plan is computed while all the new demands are inserted and all tasks already assigned to this day are kept.

Other attributes of the weekly plan are preserved through soft constraints. As patients already know their visiting time, deviations exceeding a threshold $\Delta$ are penalized. Caregivers have to carry out other activities than delivering cares. For instance, they have to prepare drugs and materials for each round or to plan some visits. Starting their round earlier or later than the original time is penalized.

\section{WEEKLY PROBLEM}

A mathematical formulation of the weekly plan problem (WP) is given in this section. The integer linear program proposed here does not provide a relevant solution tool for realistic-size instances (about 10 human resources and 400 tasks), but it provides a formal definition and is base of the solution approach of the daily plan.

This problem is closely related to the widely studied PVRP, where a customer has to be served following one of his possible patterns. The planning horizon is split into periods. A pattern is a set of periods where the customer has to be visited. For each day, routes of the fleet of vehicles based at a depot, have to visit all customers with the current day present in their selected pattern. A classical variant of PVRP includes time window constraints (on the day time) on visits. The total travel time is usually minimized over the horizon. In our case, the periods correspond to half-day and the planning horizon is one week. Route durations can not exceed half a day. In HHCP, a customer is a task and a pattern is the set of possible periods for the task; the depot is the HHC center; and the fleet of vehicles is heterogeneous as vehicles represent staff with different skills: nurse, nursing auxiliary and logistician. Each task requires a specific skill. Skills are linked by logistics tasks that can be performed by any employee. Time windows constraints can be defined for any task.

The half day split holds since lunch and team meeting take place in the HHC every midday. Those meetings are not generalized to every HHC service, and periods corresponding to complete days may be considered. Lunch breaks would have been added to the model, but they do not change the overall approach.

Assignment to staff members and routes are computed to evaluate the workload and the service date for each task, which are the output of this optimization stage. Idle times have to be planned in order to accept future demands. As new admissions often require several tasks to be performed during next days, idle times have to increase with the period, i.e., last days of the weekly plan are less loaded. Expected additional workload is modeled as an increasing linear function of the day number. Then a total target workload is defined for each period.

In the following, we propose a four-index mixed integer program (MIP) formulation of WP. The objective of WP is to minimize the total cost of transportation as well as the gap between workload and target workload for each period. Because of the size of real-world problems - hundreds of tasks - commercial MIP solvers can not solve WP and a Tabu Search algorithm is designed to provide optimized solutions.

\section{A. Mathematical formulation WP}

We present the different parameters and decision variables, followed by the objective and constraints.

\footnotetext{
- $R$ is the set of resources index by $r$.

- $T$ is the set of periods index by $t$.

- $A=\cup_{r \in R, t \in T} A(r, t)$ is the set of tasks with $A(r, t)$ the set of feasible tasks with resource $\mathrm{r}$ in period $\mathrm{t}$.

- $V=\cup_{r \in R, t \in T} V(r, t)$ where $V(r, t)=A(r, t) \cup\left\{d d_{r}\right\} \cup\left\{a d_{r}\right\}$ and $d d_{r}$ and $a d_{r}$ represent the departure and arrival depots
} 
of resource $r$, respectively.

- $t_{j k}$ for $j$ and $k \in V$, is the travel time between nodes (task or depot) $j$ and $k$. Service time of $j$ is included.

- $e_{j}$ and $l_{j}$ are the earliest (resp. latest) visit time of node $j$.

- $d_{j}$ is the service duration at node $j$.

- $E_{t}$ is the target workload for period $t$.

- $\alpha \in \mathbb{R}^{+}$is the weight of penalization.

- $M$ is a large enough number.

Three types of decision variables are used:

- $X_{j k}^{r t}$ is a binary variable equal to 1 if the resource $r$ travels directly from node $j$ to node $k$ in period $t$.

- $S_{r j}^{t}$ is the starting time of the service at node $j$ by the resource $r$ at time $t$.

- $H_{t}$ is the amount working time exceeding the target workload in period $t$.

$$
\begin{gathered}
\text { WP : } \min z=\sum_{r \in R} \sum_{j \in V} \sum_{k \in V} \sum_{t \in T} t_{j k} X_{j k}^{r t}+\alpha \sum_{t \in T} H_{t} \\
\sum_{r \in R} \sum_{t \in T: k \in A(r, t)} \sum_{j \in V(r, t)} X_{j k}^{r t}=1 \quad \forall k \in A \\
\left(X_{j k}^{r t}-1\right) M \leq S_{r k}^{t}-S_{r j}^{t}-d_{j}-t_{j k} \quad \forall r \in R, t \in T, j, k \in V(r, t) \\
\sum_{j \in V(r, t)} X_{j k}^{r t}=\sum_{j \in V(r, t)} X_{k j}^{r t} \quad \forall r \in R, t \in T, k \in A(r, t) \\
\sum_{r \in R} \sum_{j \in V(r, t)} \sum_{k \in V(r, t)} X_{k j}^{r t}\left(d_{j}+t_{j k}\right) \leq H_{t}+E_{t} \quad \forall t \in T \\
H_{t} \geq 0 \quad \forall t \in T \\
X_{j k}^{r t}=0 \quad \forall r \in R, t \in T, k \notin V(r, t) \\
X_{j k}^{r t} \in\{0,1\} \quad \forall j, k \in V, r \in R, t \in T \\
e_{j} \leq S_{r j}^{t} \leq l_{j} \quad \forall j \in V, r \in R, t \in T
\end{gathered}
$$

The objective function (1) minimizes the total routing cost in the first criterion, and minimizes the exceeding workload in the second criterion. Constraints (2) ensure that each task is assigned to a single resource and a single period. Constraints (3) compute arrival times from precedence variables. Constraints (4) ensure the continuity of routes. Constraints (5) and (6) give exceeding workload. Constraints (7) set few of infeasible assignments to zero; they can be maintained through a preprocessing step. Constraints (8) define the binary variables. Constraints (9) ensure that time windows are observed. Applied to depots constraints (9) enforce the maximal duration to routes.

\section{B. A TABU SEARCH METHOD}

Tabu Search (TS) has been proved as one of the most efficient metaheuristic approach on many vehicle routing problems, like some periodic vehicle routing problems [16], [17]. Moreover, TS requires light developments and parametrization steps.

Our TS is inspired from the Tabu Search proposed in [16] which addresses a multi-depot PVRP, and allows to explore unfeasible solutions. The general scheme of our TS is described in Algorithm 1. An initial solution is generated from a greedy list algorithm (Step 1); the list of patients is randomly generated. Neighborhoods considered at Step 2 are based on 0-1 and 1-1 exchange moves. The first move computes all possible insertion positions for each task after removing itself from its current position. The 1-1 exchange move computes all swap between pair of tasks. Note that, in our implementation, moves are considered between routes of different possible days of realization of the task. A neighbor solution is evaluated by a fitness function $g$ defined in equation 10. The last term penalizes time window constraints violation with a variable parameter $\gamma$.

$$
\begin{aligned}
g(X):=\sum_{r \in R} & \sum_{j \in V} \sum_{k \in V} \sum_{t \in T} t_{j k} X_{j k}^{r t}+\sum_{t \in T} H_{t} \\
& +\gamma \sum_{j \in V, r \in R, t \in T} \max \left(e_{j}-S_{r j}^{t} ; S_{r j}^{t}-l_{j} ; 0\right)
\end{aligned}
$$

Among all the moves evaluated, the best set of independent moves is selected at Step 3. Qualified moves have to satisfy all the constraints of the model (1)-(9) except the time window constraints. Moves are independent if their combined contribution (when moves are all performed) to the objective function is equal to the sum of independent contributions of each move. For instance two moves involving different days are independent. On a single routes moves can be independent when no time window constraints are violated before and after moving. This subset is computed by solving a general matching problem on a graph where nodes are pairs of employee and period, and edges are weighted by the best value among all neighbors involving both extreme nodes. Each node is duplicated in order to model neighbors on single routes. Independence is verified for travel time and time window costs, but not for the exceeding workload. Computational experiments show that this bias can be neglected since that case appears rarely and gives small deviations. The reader is referred to [18] for a polynomial (cubic) algorithm and [19] for an implementation in the context of a large neighborhood search for a parallel machines scheduling problem.

At Step 5, the best known solution is updated with the solution obtained by application of selected moves. As in [16], two parameters are updated at Step 6 in order to control the divergence of the exploration. The main one is the tabu list. For each task involved in a selected move, the origin attribute (day, employee, position in the sequence) of the task is forbidden for a fix number of iterations. In that way, one can expect to avoid loops on a small set of solutions. Each position is also associated with an aspiration value, which is defined as the cost of the best feasible solution found with that position. Thus, a neighbor solution $\bar{X}$ of the current solution $X$ can be considered only when: (1) all new positions which are not in $X$ but in $\bar{X}$, are non-tabu, or (2) $\bar{X}$ is feasible and $z(\bar{X})$ is less than the aspiration values of these positions. Steps 2-7 are repeated until a limited number of iterations is reached. 
Time complexity of both neighborhoods exploration is cubic, since the evaluation of the violation of time window constraints can require a linear time-complexity algorithm. This worst case is not met in general, since evaluated moves only involve qualified employees and possible visiting days.

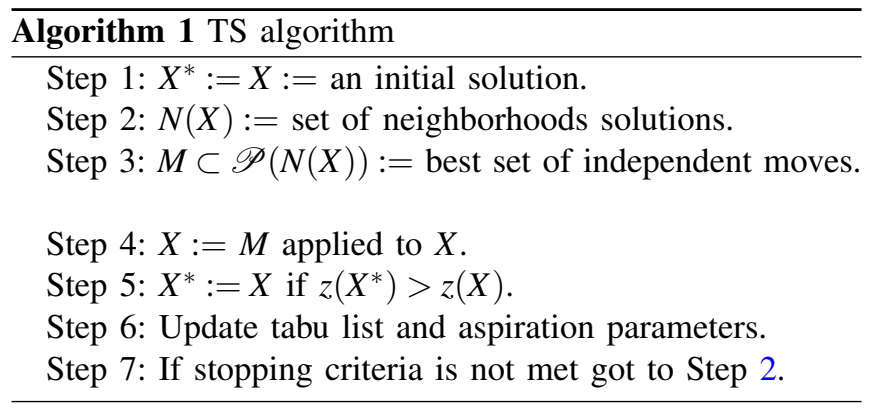

\section{DAILY PROBLEM}

Some unknown demands when the weekly plan has been computed, have to be inserted each day. We assume that all daily demands are known before each day. Insertion of new demands have to preserve as far as possible the time schedule of the weekly plan. Assignment to staff members is left as a free decision variable. We expect that the original solution is obviously close to the optimal solution after insertion, since the objective function penalizes deviations from the original solution. The size of the daily problem allows us to use a branch-and-cut solver to improve the original solution. The integer linear formulation of the daily plan problem (DP) and the MIP-based heuristic approach are described in the two next sections.

\section{A. Mathematical formulation DP}

In this problem, new parameters and variables are introduced. Constraints (9) on time windows are replaced by a piecewise linear penalty function on service time deviation. For each task $j$, the function is decreasing until $A_{j}$, equal to zero between $A_{j}$ and $B_{j}$ and decreasing after $B_{j}$, where $A_{j}$ is set to a quarter before the service time of the weekly plan solution and $B_{j}$ a quarter after. Early starting time and late ending times of routes of each resource are also penalized by linear functions.

In the following, we propose a four-index MIP formulation of the daily plan problem (DP). The objective of the DP is to minimize the total cost of transportation and penalties on earliness and lateness compared with the initial plan. DP is based on WP. Constraints related to the target workload are removed and new constraints are added. Additional parameters and variables are listed below.

- $\left[A_{j}, B_{j}\right]$ is the new soft time window defined for task $j$. - $\left[T G_{r}^{t}, T D_{r}^{t}\right]$ are the starting and ending time of the route of resource $r$ at period $t$ in the initial plan.

- $E A_{j}$ and $E B_{j}$ measure the earliness (resp. tardiness) of task $j$.

- $A v_{r}^{t}$ and $R v_{r}^{t}$ measure the earliness (resp. tardiness) of route $r$ at period $t$.
- $\mu$ and $\delta$ are the penalization weights of both types of time deviation from the weekly plan.

$$
\begin{gathered}
\text { DP : } \min Z=\sum_{r \in R} \sum_{t \in T} \sum_{j \in V(r, t)} \sum_{k \in V(r, t)} t_{j k} X_{j k}^{r t} \\
+\mu \sum_{j \in A} E A_{j}+\mu \sum_{j \in A} E B_{j} \\
+\delta \sum_{r \in R} \sum_{t \in T}\left(T G_{r}^{t}-A v_{r}^{t}\right)+\delta \sum_{r \in R} \sum_{t \in T}\left(R v_{r}^{t}-T D_{r}^{t}\right)
\end{gathered}
$$

subject to constraints (2), (3), (4), (7), (8) and

$$
\begin{gathered}
A v_{r}^{t} \leq T G_{r}^{t} \quad \forall r \in R, t \in T \\
A v_{r}^{t} \leq S_{r d d_{r}}^{t} \quad \forall r \in R, t \in T \\
R v_{r}^{t} \geq T D_{r}^{t} \quad \forall r \in R, t \in T \\
R v_{r}^{t} \geq S_{r a d_{r}}^{t} \quad \forall r \in R, t \in T \\
S_{r j}^{t}+E A_{j} \geq A_{j} \quad \forall r \in R, t \in T, j \in A(r, t) \\
S_{r j}^{t}-E B_{j} \leq B_{j} \quad \forall r \in R, t \in T, j \in A(r, t) \\
S_{r j}^{t} \geq 0, \quad \forall j \in A, r \in R, t \in T \\
E A_{j}, E B_{j} \geq 0 \quad \forall j \in A \\
A v_{r}^{t}, R v_{r}^{t} \geq 0 \quad \forall r \in R, t \in T
\end{gathered}
$$

Constraints (12)-(13) compute the minimum between solution starting time and the initial one. Constraints (14)-(15) do the same for ending route times. Earliness and lateness of service times are computed in constraints (16) and (17), respectively.

\section{B. MIP-based heuristic}

Several researchers tried to exploit the efficiency of solvers and convergence properties of exact methods, based for instance on linear programming or constraint programming, to design heuristic or metaheuristic algorithms. Such approaches form a part of matheuristics. For further details, the reader is sent to two surveys on matheuristics for routing problems [20] and [21]. Such approaches can perform well on improving already good solutions when classical metaheuristics fail to get improvements. Another interesting feature gives robustness to such schemes, as it is easy to add side constraints using solver interfaces.

The heuristic proposed here follows this line of research by applying intensive search on series of sub-problems.

In a first phase, a list algorithm inserts all uncovered tasks in the solution obtained by solving WP. A best insertion only based on travel time minimization is used here. Finally, all tasks of the current day are covered in what we call initial solution.

The day of visit of nodes is kept from the initial solution, but the period can change to morning or evening. Our heuristic considers one DP for each pair of periods in the same day.

From the initial solution, some sub-sequences of routes are fixed. The MIP DP is run with additional constraints 
that enforce selected sub-sequences, as in equation 21 . In other words, sub-sequences becomes nodes in the routing problem. Sub-sequences can therefore be assigned to any resource with required skills. A limited number of subsequences are randomly generated. This number is defined by the computational capacity of the solver to provide optimized solutions within a time limit. That scheme allows us to consider complex combinations of basic moves.

$$
\sum_{r \in R} \sum_{t \in T} X_{j k}^{r t}=1, \quad \text { if } j \text { precedes } k \text { in the sub-sequence }
$$

\section{COMPUTATIONAL EXPERIMENTS}

This section reports the results of a series of computational experiments for evaluation of our two step approach and of our modeling.

To the best of our knowledge no paper deals with weekly planning of home health care, including dynamic demands and detailed traveling workload. No benchmark instances exist to test our approach. We built instances in the following way. Ratios of type of tasks are set from historical data of the HHC service in Sallanches (France). We derive parameters from data and experience of that HHC center, in order to generate sets of instances. One half require nurses and have durations between 10 and 30 minutes, $20 \%$ require nursing auxiliary with duration between 30 and 60 and other tasks concern logistics and have durations of 5 minutes (drug delivery in general). Employees are distributed with $50 \%$ of nursing auxiliary, $40 \%$ of nurses and $10 \%$ of logisticians. Task nodes are uniformly generated in a square $100 \times 100$ and travel times are set as euclidean distances. Only one depot is considered as the HHC center and is placed in the middle of the square.

Algorithms have been coded in $\mathrm{C}++$ and run on a 2.4 $\mathrm{GHz}$ processor. IBM Ilog Cplex 12.5 software is used to run the branch-and-cut algorithms in the second step heuristic. The complete models WP and DP have also been run on some instances but Cplex fails to find any feasible solution even for problems on 3 periods, 5 resources and less than 60 tasks. Ten instances have been generated with 10 resources (4 nurses, 5 nursing-auxiliaries and 1 logistician), 380 tasks (known before the week) and 10 periods of 4 hours (2 per day). All objectives are expressed in minutes and their weights in objective functions $(\alpha, \mu$ and $\delta$ ) are set to 1 .

In Table I, we show the results obtained by the tabu search algorithm. For each instance, 3 runs of 1000 iterations (few improvements happen after 500 iterations) have been performed. Average computing times and objective values (total working time, gap with the target working time and time windows constraints violation) can be found in the last three columns, given in minutes. Values obtained for the 10 instances are aggregated on each row by average, minimum and maximum value, respectively. The total workload (about 5 hours per day, since the value in column work is given in minutes and concerns 10 employees for 5 days) seems low, but some time is required for next arrivals and employees also have to deal with other activities in the center. Total extra working time is large but it is computed from a rough estimation (the tasks duration plus one half of the distance to the depot). The most important behavior to notice is the decreasing workload obtained in Table II, where one can see the impact of the objective on expected workload. Although time windows are defined as hard constraints in WP, our tabu search is not always able to find feasible solutions (it may not exist). The total number of minutes of deviations over the week is given in column TW. Minimal and maximal values of Table I indicate that the solutions obtained are stable on the 10 instances generated. But in Table II, the gap between minimal and maximal planned workload on the weekly basis can vary by more than $40 \%$. It means that depending on the geographical distribution of patients, the algorithm let more or less idle time on different days.

TABLE I

Results of Tabu search on the weekly plan

\begin{tabular}{|l|l|l|l|l|}
\hline & cpu (s.) & work & extra work. & TW \\
\hline avg & 14.0 & 14355.3 & 3019.1 & 86.7 \\
min & 13 & 13007 & 2076 & 0 \\
$\max$ & 16 & 14898 & 3446 & 267 \\
\hline
\end{tabular}

TABLE II

Daily workload in the weekly plan

\begin{tabular}{|l|l|l|l|l|l|}
\hline & Mon. & Tue. & Wed. & Thu. & Fri. \\
\hline avg & 4015.9 & 3092.1 & 2735.3 & 2372.4 & 2139.6 \\
$\min$ & 3600 & 2431 & 2385 & 1765 & 1521 \\
$\max$ & 4721 & 3714 & 3383 & 2663 & 2822 \\
\hline
\end{tabular}

New demands are generated for each day of the week to reach 475 tasks over the week. It matches real data where about $20 \%$ of tasks are revealed during the week. Our algorithm has been run three times on each instance following three different policies. The initial model (P1) is compared to two other policies: in (P2) the weekly plan does not allow flexibility on the period for task realization (the preferred period is enforced); in (P3) the step penalization based on expected demand is discarded. Ten iterations (one iteration requires around 1 minute) of our second heuristic are performed for each day (a pair of periods). Average results obtained at the first and second phase are listed for each policy in Table III. The final total costs (sum over five days) and different objectives values are given in minutes (earliness and tardiness on tasks service, and on round of resources). Results obtained for two instances are in the two first sets of three rows. The last three rows are average values over the 10 instances. The working time obtained after the first phase for $\mathrm{P}(1)$ is not minimal compared to other policies, but the final total cost after the second phase is minimal for $\mathrm{P}(1)$. Thus, on this set of instances the policy $\mathrm{P}(1)$ allows to provide more robust solutions than $\mathrm{P}(2)$ and $\mathrm{P}(3)$ considering a combination of five objectives.

Even if the total working time is higher in the first phase solution for policy $\mathrm{P}(1)$, it allows to reach, in average, a better solution after the insertion of new demands after the second phase. 
TABLE III

Comparison of three policies

\begin{tabular}{|l|l|l|l||l|l|l|l|l|l|}
\hline & \multicolumn{3}{|c|}{ first phase } & \multicolumn{5}{c|}{ second phase } \\
\hline Pol. & work & ex. work & TW & Tot. cost & travel & task earl. & task tard. & res. earl. & res. tard. \\
\hline $\mathrm{P}(1)$ & 15129 & 3468 & 96 & 15867 & 10027 & 143 & 2733 & 54 & 2874 \\
$\mathrm{P}(2)$ & 14825 & 3166 & 31 & 16609 & 10461 & 66 & 2736 & 88 & 3223 \\
$\mathrm{P}(3)$ & 14586 & 0 & 28 & 16423 & 10457 & 81 & 2877 & 18 & 2954 \\
\hline $\mathrm{P}(1)$ & 15274 & 3730 & 97 & 15102 & 10116 & 93 & 2184 & 63 & 2623 \\
$\mathrm{P}(2)$ & 14872 & 0 & 69 & 16310 & 10838 & 89 & 2377 & 101 \\
$\mathrm{P}(3)$ & 14768 & 3224 & 56 & 16387 & 10378 & 196 & 2613 & 66 & 2874 \\
\hline $\mathrm{P}(1)$ & 14241 & 2939 & 69 & 15328 & 9786 & 87 & 2352 & 0 & 3114 \\
$\mathrm{P}(2)$ & 14981 & 3677 & 105 & 15932 & 10457 & 96 & 2581 & 26 & 2699 \\
$\mathrm{P}(3)$ & 14258 & 14258 & 85 & 15664 & 9912 & 99 & 2610 & 40 & 2745 \\
\end{tabular}

\section{CONCLUSIONS}

This paper investigates a human resources planning problem in HHC services including some specific characteristics of the French system.

Our problem is related to highly complex vehicle routing problems including PVRP (Periodic Vehicle Routing Problem) and PVRPTW (Periodic Vehicle Routing Problem with Time Window). It can be easily proved that our problem is strongly NP-hard because it covers classical VRP problems as a particular case. Even for a small-sized HHC service, the literature on periodic vehicle routing problems shows that problems with at least 400 hundreds nodes become intractable for state-of-the-art exact approaches. Thus we design a heuristic able to provide solutions to two related problems: the weekly and the daily planning where new demands are revealed. Despite the lack of information about the position of demands, our computational experiments show that the planner can take advantage of a rough estimation of the uncertain demands and an accurate routing estimation. This work can be continued following two ways. First, the algorithms can be improved, especially the second phase. It seems that a faster heuristic can be applied before running the second phase matheuristic. The Tabu Search of the first phase can be easily adapted to this problem. A second line of work deals with further evaluations of this approach on real-world applications and benchmarking with other applications.

\section{ACKNOWLEDGMENT}

This research is founded by Labex IMobS'3 (ClermontFerrand, France) for providing a PhD Student fellowship for the first author. This work was developed in the Centre for Biomedical and Healthcare Engineering at Ecole Nationale Superieure des Mines de Saint-Etienne (France).

\section{REFERENCES}

[1] Coppi, A., Detti, P., \& Raffaelli, J. (2013). A planning and routing model for patient transportation in health care. Electronic Notes in Discrete Mathematics, 41, 125-132.

[2] Bertels, S., \& Fahle, T. (2006). A hybrid setup for a hybrid scenario: combining heuristics for the home health care problem. Computers \& Operations Research, 33(10), 2866-2890.

[3] Lanzarone, E., Matta, A., \& Scaccabarozzi, G. (2010). A patient stochastic model to support human resource planning in home care. Production Planning and Control, 21(1), 3-25.
[4] Eveborn, P., Flisberg, P., \& Rönnqvist, M. (2006). LAPS CARE: an operational system for staff planning of home care. European Journal of Operational Research, 171(3), 962-976.

[5] Nickel, S., Schrder, M., \& Steeg, J. (2012). Mid-Term and Short-Term Planning Support for Home Health Care Services. European Journal of Operational Research, 219, 574?587.

[6] Bard, J. F., Shao, Y., \& Wang, H. (2013). Weekly scheduling models for traveling therapists. Socio-Economic Planning Sciences, 47(3), 191-204.

[7] Campbell, A. M., \& Wilson, J. H. (2014). Forty years of periodic vehicle routing. Networks, 63(1), 1097-0037.

[8] Baldacci, R., Bartolini, E., Mingozzi, A., \& Valletta, A. (2011). An exact algorithm for the period routing problem. Operations research, 59(1), 228-241.

[9] Gulczynski, D., Golden, B., \& Wasil, E. (2011). The period vehicle routing problem: New heuristics and real-world variants. Transportation Research Part E: Logistics and Transportation Review, 47(5), 648-668.

[10] Hemmelmayr, V.C., Doerner, K.F., \& Hartl, R.F. A variable neighborhood search heuristic for periodic routing problems. European Journal Operation Research, 195, 791 ?802.

[11] Vidal, T., Crainic, T. G., Gendreau, M. , Lahrichi, N., \& Rei, W. (2012) A hybrid genetic algorithm for multidepot and periodic vehicle routing problems. Operation Research, 60, 611?624.

[12] Wen, M., Cordeau, J. F., Laporte, G., \& Larsen, J. (2010). The dynamic multi-period vehicle routing problem. Computers \& Operations Research, 37(9), 1615-1623.

[13] Mourgaya, M., \& Vanderbeck, F. (2007). Column generation based heuristic for tactical planning in multi-period vehicle routing. European Journal of Operational Research, 183(3), 1028-1041.

[14] Steeg, J., \& Schröder, M. (2008). A hybrid approach to solve the periodic home health care problem. In Operations Research Proceedings 2007 (pp. 297-302). Springer Berlin Heidelberg.

[15] Cappanera, P., \& Scutell, M. G. (2013). Home Care optimization: impact of pattern generation policies on scheduling and routing decisions. Electronic Notes in Discrete Mathematics, 41, 53-60.

[16] Cordeau, J. F., Gendreau, M., \& Laporte, G. (1997). A tabu search heuristic for periodic and multi-depot vehicle routing problems. Networks, 30(2), 105-119.

[17] Liu, R., Garaix, T., \& Xie, X. (to appear). Hybridization of Tabu Search with Feasible and Infeasible Local Searches for Periodic Home Health Care Logistics. Omega.

[18] Gabow, Harold N. (1976). An Efficient Implementation of Edmonds' Algorithm for Maximum Matching on Graphs. Journal of the ACM, 23(2), 221-234.

[19] Della Croce, F., Garaix, T., \& Grosso, A. (2012). Iterated local search and very large neighborhoods for the parallel-machines total tardiness problem. Computers \& Operations Research, 39(6), 1213-1217.

[20] Doerner, K. F., \& Schmid, V. (2010). Survey: matheuristics for rich vehicle routing problems. In Hybrid Metaheuristics. (pp. 206-221). Springer Berlin Heidelberg.

[21] Archetti, C., \& Speranza, M. G. (2013). A survey on matheuristics for routing problems. Tech. Report. Univ. Brescia. 